Animal Reproduction Research Institute (ARRI),

Giza-Egypt.

\title{
EVALUATION OF ROSE BENGAL TEST, GEL IMMUNODIFFUSION WITH NATIVE HAPTEN, AND INDIRECT ELISA IN DIAGNOSIS OF BRUCELLOSIS IN INFECTED AND VACCINATED SHEEP
}

(With 2 Tables)

\section{By}

\author{
M.M. BASSIONY, K.A. ABOU GAZIA, \\ M.W. ABD AL-AZEEM* and I.G.A. IBRAHIM \\ * Department of Microbiology, Faculty of Vet. Med., SouthValley Univ.
}

(Received at 26/1/2010)

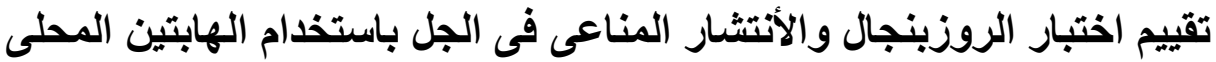

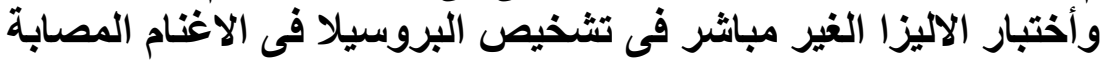
والمحصنة بالبروسيلا فئمس الميرو

$$
\begin{aligned}
& \text { محد محمد بسبيونس ، خالد عبد السمبع ابوجازية }
\end{aligned}
$$

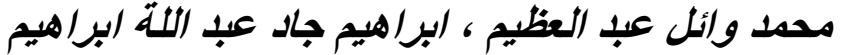

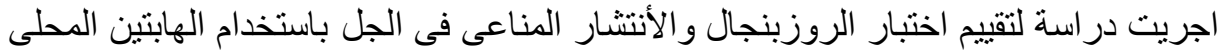

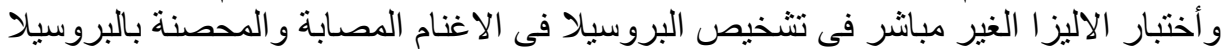

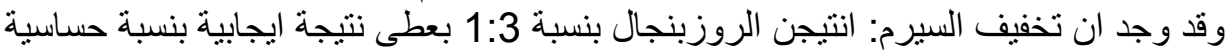

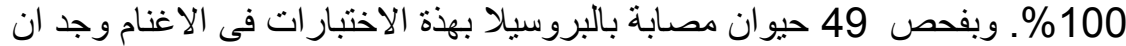

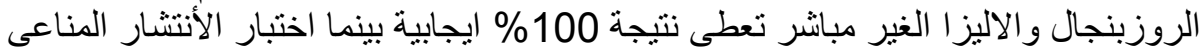

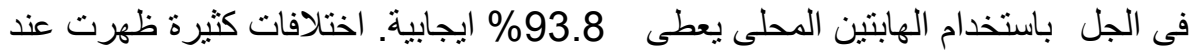

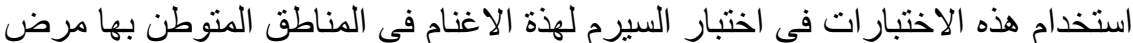

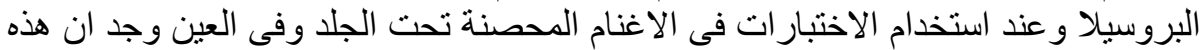

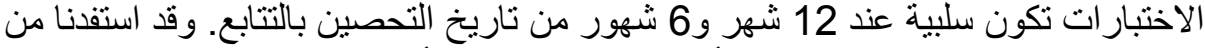

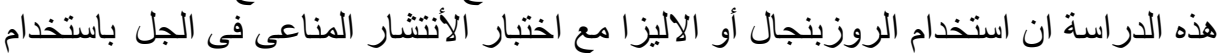

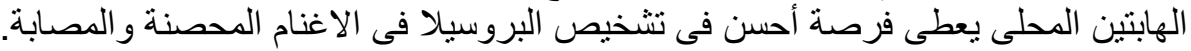

\section{SUMMARY}

Three serological assays were evaluated for the diagnosis of brucellosis in sheep: the Rose Bengal plate test as a screening test (RBPT), agar gel immunodiffusion with Native Hapten test (AGID-NH) and an indirect enzyme-linked immunosorbent assay (iELISA). For optimal sensitivity, 
RBPT had to be used with sera: antigen at a 3:1 dilution. With the sera from B. melitensis culture-positive sheep, the sensitivity was $100 \%$ for RBPT, and iELISA; while, it was $93.8 \%$ for AGID-NH test. On the other hand specificity was $100 \%$ when testing the sera from 145 brucella-free sheep. Larger discrepancies among the results of the serological tests were obtained with sera from sheep of areas where brucellosis is endemic. Sheep vaccinated with B. melitensis Rev.1 subcutaneously and conjunctively showed negative result in all tests after twelve and six months respectively. The proportion of sheep giving a positive reaction after vaccination decreased faster in AGID-NH test than in other tests. The using of RBPT or ELISA with combination with AGID-NH is the best choice for diagnosis of infected and vaccinated sheep.

Key words: Brucella, iELISA, AGID-NH

\section{INTRODUCTION}

Brucellosis is an infectious disease of worldwide importance in domestic ruminants, and the causative bacteria are transmitted to humans through contact with infected livestock or by consumption of contaminated dairy products. Because of the economic importance of cattle in developed countries, means for $B$. abortus diagnosis and prophylaxis have been widely investigated (Alton et al., 1988; Nicoletti, 1990; Plommet and Fensterbank, 1984; Zundel et al., 1992) and several serological tests developed for cattle brucellosis have been found useful for the diagnosis of B. melitensis infection in sheep (Alton, 1990; DiazAparicio et al., 1993; Jimenez de Bagues et al., 1992).

The control of sheep brucellosis is usually based on vaccination, serological testing, and culling the positive. Until now, the best vaccine available has been the smooth $B$. melitensis Rev.1 strain. Although this strain is useful, it does not give $100 \%$ protection, and it induces a strong antibody response to the S lipopolysaccharide (S-LPS).

Since S-LPS is the most relevant antigen in conventional serological tests such as the Rose Bengal plate test (RBPT) and the complement fixation test (CFT) (Alton et al., 1988), it is not surprising that Rev.1 vaccination interferes with serological diagnosis. Even so, conventional serological diagnosis requires the use of screening and confirmatory tests such as RBPT and CFT, respectively. However, the use of these two tests does not result in $100 \%$ sensitivity and specificity (Blasco, 1994 b). To reduce these restrictions in the use of the vaccine 
and to facilitate serological diagnosis, alternative assays have been investigated, including tests that detect antibodies to proteins (D1'az et al., 1984; Zygmunt, et al., 1994; Debbar et al., 1995; Letesson, et al., $1997)$ and to the S-LPS-related native hapten $(\mathrm{NH})$ polysaccharide (Blasco et al., 1984 a; Jime'nez de Bagu“e's et al., 1992; D1'az-Aparicio et al., 1993; D1'az-Aparicio et al., 1994).

The aim of the present work was to standardize the most used conventional test (i.e., RBPT) for sheep brucellosis, to compare the AGID-NH test with the iELISA using control sera from infected and brucella-free sheep and sera from sheep from areas where brucellosis is endemic; and to evaluate the interference of the postvaccinal serological response in the previously mentioned tests.

\section{MATERIALS and METHODES}

\section{A- Sera separated from the collected blood samples of the following animals were used:-}

- Group I: B. melitensis-infected sheep. Forty nine sheep with field vaccination had not been used. No previous selection of the animals was made on the basis of any serological test.

- Group II: Vaccinated sheep. A young sheep (3 months old) were vaccinated subcutaneously (59 animals) and conjunctively (34 animals) with $10^{9} \mathrm{CFU}$ of $B$. melitensis Rev.1 and blood samples were collected every 2 months after subcutaneous and conjunctival vaccination till be negative serologically.

- Group III: Brucella-free sheep. One hundred forty five sheep were from two flocks free of brucellosis in which vaccination had not been performed.

- Group IV: non-vaccinated sheep from areas in which brucellosis is endemic. Two hundred thirty sheep were from an area where brucellosis is endemic and no vaccine used.

B- Serological tests: (1) RBPT was performed with a 3:1 of proportion serum to antigen for optimal sensitivity (Blasco et al., 1994).

(2) Agar gel immunodiffusion test for detecting NH-precipitating antibodies (AGID-NH) was performed with $1 \%$ Noble agar (Difco Laboratories, Detroit, Mich.) gels in 10\% NaCl-0.1 M NaOH-H3BO4 (pH 8.3) with $20 \mathrm{ul}$ of serum and the antigen wells set $3 \mathrm{~mm}$ apart. The antigen was an NH (Brucella Lab. CITA, Zaragoza, Spain) rich B.melitensis $16 \mathrm{M}$ hot-water extract in which the $\mathrm{NH}$ precipitation ring 
is characteristic (D1'az et al., 1981; Moreno et al., 1981; D1'az et al., 1984; Arago'n et al., 1996).

(3) iELISA was performed with a crude B.melitensis S-LPS preparation (D1'az et al., 1981; Jime'nez de Bagu“e's et al., 1992; Arago'n et al., 1996; Alonso-Urmeneta et al., 1998) and peroxidase-conjugated protein $\mathrm{G}$, and the results were expressed as the percentage of the optical density with respect to a strongly positive control serum (Jime'nez de Bagu“e's et al., 1992; D1'az-Aparicio et al., 1994; Alonso-Urmeneta et al., 1998).

The sensitivity and specificity of the tests were calculated with respect to the infected and brucella-free groups as described by Jones et al. (1973).

\section{RESULTS}

The sera from the B.melitensis infected and brucella-free animals showed optimal sensitivity with 1:50 of the serum dilution, with these sera, the iELISA completely discriminated the sera from the brucellafree and B.melitensis-infected populations $(100 \%$ sensitivity and specificity). The RBPT had performed as currently recommended failed to detect 4 of the 49 infected sheep (91.8\% sensitivity). In contrast, when the proportion of serum was increased ( $25 \mu \mathrm{l}$ of antigenic suspension and $75 \mathrm{ul}$ of serum), RBPT gave no false-negative results (Table 1) and showed $100 \%$ sensitivity. The two tests were more sensitive than the AGID-NH test (93.8\%). The AGID-NH test and RBPT showed 100\% specificities for sera from brucella-free animals.

On the other hand, the results obtained with sera from Sheep from an area in which brucellosis is endemic (Table 1) showed larger discrepancy than the results obtained with sera from infected and free sheep. The RBPT and iELISA showed sensitivity of 35.6\% and 33.0\% respectively, while the AGID-NH test showed a sensitivity of only $18.2 \%$.

Table 2 shows the interference of the post-vaccinal serological response in the above serological tests. The specificities of the tests (i.e., the percentage of vaccinated animals that tested negative and, therefore, would not be misdiagnosed as infected) varied depending upon the route of vaccination. In subcutaneous route, the AGID-NH test was $100 \%$ specific at six months following vaccination, while the RBPT and iELISA showed specificity of $32.8 \%$ and $24.1 \%$ respectively. On the 
other hand, the AGID-NH test had a faster specificity of $100 \%$ at four months post-vaccination using the conjunctival rout. The RBPT and iELISA had specificity of $85.3 \%$ and $76.5 \%$ respectively at the same period.

Table 1: Results of serological tests of sera from infected sheep and sheep of endemic areas.

\begin{tabular}{|c|c|c|c|c|c|}
\hline \multicolumn{2}{|c|}{ Tested Group } & \multirow[t]{2}{*}{ Status } & \multicolumn{3}{|c|}{$\begin{array}{c}\text { TEST } \\
\text { No. of serum samples positive in }\end{array}$} \\
\hline $\begin{array}{c}\text { Group } \\
\text { No. }\end{array}$ & $\begin{array}{c}\text { Animal } \\
\text { No. }\end{array}$ & & RBPT & ELISA & AGID-NH \\
\hline I & 49 & Infected & 49 & 49 & 44 \\
\hline \multicolumn{2}{|c|}{ Sensitivity } & & $100 \%$ & $100 \%$ & $93.8 \%$ \\
\hline II & 230 & \multirow[t]{2}{*}{ Endemic } & 82 & 76 & 42 \\
\hline \multicolumn{2}{|c|}{ Sensitivity } & & $35.6 \%$ & $33.0 \%$ & $18.2 \%$ \\
\hline
\end{tabular}

Table 2: Specificities of serological tests for brucellosis for sera from B.melitensis Rev1-vaccinated sheep

\begin{tabular}{|c|c|c|c|c|c|c|}
\hline \multirow{2}{*}{$\begin{array}{c}\text { Age of } \\
\text { vaccination } / \mathrm{m}\end{array}$} & \multirow[t]{2}{*}{ Rout } & \multirow{2}{*}{$\begin{array}{l}\text { Sampling } \\
\text { /month(b) }\end{array}$} & \multirow{2}{*}{$\begin{array}{l}\text { No. of } \\
\text { Sheep }\end{array}$} & \multicolumn{3}{|c|}{$\%$ Specificity of (a) } \\
\hline & & & & RBPT & AGID-NH & iELISA \\
\hline \multirow{6}{*}{3} & \multirow{6}{*}{ 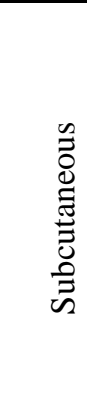 } & 2 & 59 & 8.5 & 30.5 & 3.4 \\
\hline & & 4 & 58 & 15.5 & 82.8 & 10.3 \\
\hline & & 6 & 58 & 32.8 & 100 & 24.1 \\
\hline & & 8 & 55 & 63.6 & 100 & 58.1 \\
\hline & & 10 & 48 & 89.6 & 100 & 87.5 \\
\hline & & 12 & 47 & 100 & 100 & 100 \\
\hline \multirow{3}{*}{3} & \multirow{3}{*}{ 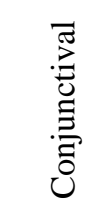 } & 2 & 34 & 32.4 & 88.2 & 26.5 \\
\hline & & 4 & 34 & 85.3 & 100 & 76.5 \\
\hline & & 6 & 34 & 100 & 100 & 100 \\
\hline
\end{tabular}

$\mathrm{a}=$ Specificity is defined as the percentage of vaccinated animals that are negative in a given test. $b=$ Number of months after vaccination 
In the present work, the above tests have been evaluated using sera from sheep with brucellosis proved by bacteriological isolation of B.melitensis biotype 3 from their tissues and milk samples. The results show that they are all potentially useful for the diagnosis of $B$. melitensis infection in sheep. However, the RBPT had to be modified by increasing the serum/antigen ratio to reach $100 \%$ sensitivity. This confirms (Blasco et al., 1994 a) that present guidelines for the standardization of the RBPT are not adequate for sheep and goat brucellosis. Therefore, the lower sensitivity reported before for RBPT (Falade, 1978; Waghela et al., 1980) is possibly due to the use of sera from animals of unknown infectious status and / or, the use of the standard method.

The sera from the B. melitensis infected and brucella-free sheep showed that iELISA yielded optimal sensitivity and specificity with the 1:50. Serum dilution. With these sera, the iELISA completely discriminated the sera from the Brucella-free and B. melitensis-infected populations (100\% sensitivity and specificity). The assay described here with S-LPS is not essentially different from some indirect ELISAs used for animal brucellosis (Alonso-Urmeneta et al., 1988; Jime'nez de Bagu"e's et al., 1992; Nielsen et al., 1988; Wright and Nielsen, 1990). But several methodological aspects deserve further comments. It was found that the protein $\mathrm{G}$ conjugate reduced the reactivity of the negative sera (i.e., it increased the ELISA specificity). In cattle brucellosis, Wright and Nielsen (1990) have found that an anti-IgGl monoclonal conjugate increases the specificity of the indirect ELISA (with S-LPS) with respect to conjugates prepared with polyclonal antibodies to $\operatorname{IgG}$ heavy and light chains or with a monoclonal antibody to the light chain.

When sera from unvaccinated sheep in areas where brucellosis is endemic were tested, a large disagreement was found between the AGID-NH test and RBPT. There is evidence that sera with specific Ig M but without significant levels of Ig G are positive in RBPT and negative in AGID-NH test (Alonso-Urmeneta et al., 1988). Thus, those discrepant results could correspond to animals that were in the early stages of the host-parasite interaction before the infection was (or was not) established.

Since live attenuated vaccines are powerful tools in the eradication of brucellosis, the interference of the postvaccinal response in the serological diagnosis is a major problem and should be considered whenever serological tests are evaluated. The specificities of all tests were generally higher when the sera of conjunctively vaccinated sheep 
were tested (Jime'nez de Bagu“e's et al., 1992). Jones et al. (1973) reported that the RBPT test and CFT become negative by the 4th month after subcutaneous vaccination of goats with $10^{9} \mathrm{CFU}$ of Rev.1, and the more protracted positive serological response found in our work is likely to be due to the use of a modified RBPT. Also, our results suggest that ELISA with S-LPS would not outperform RBPT after subcutaneous vaccination. Jones et al. (1973) also observed that vaccination with a much reduced dose $\left(5 \times 10^{4} \mathrm{CFU}\right)$ of Rev. 1 shortened the postvaccinal response to less than 2 months. However, there is evidence that vaccination with reduced doses confers a less solid immunity (Elberg, 1981), and therefore, the conjunctival route (Fensterbank et al., 1987; Zundel et al., 1992) seems a better alternative. The results presented here confirm for RBPT (Fensterbank et al., 1987), and illustrate for iELISA, and the AGID-NH tests, that the serological response after vaccination of sheep is considerably reduced by this route, with the AGID-NH test as the test that become negative faster. This reduction of the postvaccinal serological response by the use of the conjunctival route has also been shown in cattle (B. abortus 19 vaccine) and sheep (B. melitensis Rev.1 vaccine) ((Jime'nez de Bagu“e's et al., 1992).

It is clear that a relatively simple test such as the AGID-NH test was specific in diagnosis of brucellosis in sheep. Immunochemical studies have shown that the NH and the O-polysaccharide of the S-LPS (which is the serologically relevant section of S-LPS) have similar structures and epitopic densities (Arago' et al., 1996; D1'az-Aparicio et al., 1993). In fact, antibodies to the NH can be absorbed with S-LPS (Alonso-Urmeneta et al., 1988). However, precipitation tests with S-LPS do not show the sensitivity and specificity of similar tests performed with NH (D1'az et al., 1984; D1'az et al., 1981; Moreno et al., 1981), although NH and S-LPS yield similar results in both iELISA (AlonsoUrmeneta et al., 1998; D1'az-Aparicio et al., 1994) and passive hemagglutination (Alonso-Urmeneta et al., 1988).

To explain these apparently contradictory observations, we have proposed (Alonso-Urmeneta et al., 1988) that the higher specificities of the precipitation tests with $\mathrm{NH}$ result from two sets of factors. First, the dispersed state of the low-molecular-weight NH (Arago'n et al., 1996) in solution, as opposed to the highly aggregated S-LPSs, may be relevant in explaining their different behavior in precipitation tests. Second, if lowaffinity antibodies are predominant after vaccination, the higher threshold affinity of precipitation tests compared to that of iELISAs (Peterfy et al., 1998) may explain why NH fails to react with sera from 
vaccinated animals in the former but not in the latter assay (Gabriela et al., 2009).

The results of this work have practical implications concerning the use of the tests evaluated. In the absence of vaccination, the iELISA and the much less sophisticated RBPT (standardized and performed as described (Blasco et al., 1994 a) should be the tests of choice because of their very high sensitivities. When vaccination was implemented, no single test simultaneously afforded $100 \%$ sensitivity and specificity (Dabdoob et al., 2000).

However, screening with either the iELISA or the RBPT followed by confirmation by means of the AGID-NH test would afford the best combination of sensitivity and specificity, the latter seems to be the simplest choice for the diagnosis of sheep brucellosis when Rev.1 vaccination is implemented.

\section{ACKNOWLEDGMENT}

We have to express our grateful to all who give a hand to finish this work:

- Spanish Agency for International Development Cooperation. (AECID)

- J. M. Blasco and C. Marin, Brucella Lab. CITA, Zaragoza Spain.

\section{REFERENCES}

Alonso-Urmeneta, B.; Marı'n, C.; Arago'n, V.; Blasco, J.M.; Di'az, R. and Moriyo'n, I. (1998): Evaluation of lipopolysaccharides and polysaccharides of different epitopic structures in the indirect enzyme-linked immunosorbent assay for diagnosis of brucellosis in small ruminants and cattle. Clin. Diagn. Lab. Immunol. 5: 749-754.

Alonso-Urmeneta, B.; Moriyo'n, I.; Di'az, R. and Blasco, J.M. (1988): Enzymelinked immunosorbent assay with Brucella native hapten polysaccharide and smooth lipopolysaccharide. J. Clin. Microbiol. 26: 2642-2646.

Alton, G.G. (1990): Brucella melitensis, p. 383-409. In K. Nielsen and J.R. Duncan (ed.), Animal brucellosis. CRC Press, Inc., Boca, Raton, Fla.

Alton, G.G.; Jones, L.M.; Angus, R.D. and Verger, J.M. (1988): Techniques for the brucellosis laboratory. Institut National de la Recherche Agronomique, Paris. 
Arago'n, V.; Dl'az, R.; Moreno, E. and Moriyo'n, I. (1996): Characterization of Brucella abortus and Brucella melitensis native haptens as outer membrane O-type polysaccharides independent from the smooth lipopolysaccharide. J. Bacteriol. 178: 1070-1079.

Blasco, J.M.; Di'az, R.; Moriyo'n, I. and Salvo, M.D. (1984,a): Evaluation of a radial immunodiffusion test for diagnosing brucellosis in sheep and its possible value for differentiating infected from Brucella melitensis REV. 1 vaccinated sheep. Dev. Biol. Stand. 56: 507-511.

Blasco, J.M.; Garin-Bastuji, B.; Marl'n, C.M.; Gerbier, G.; Fanlo, J.; Jime'nez de Bagu"e's, M.P. and Cau, C. (1994,b): Efficacy of different Rose Bengal and complement fixation antigens for the diagnosis of Brucella melitensis infection in sheep and goats. Vet. Rec. 134: 415-420.

Blasco, J.M.; Marı'n, C.; Jime'nez de Bague's, M.; Barbera'n, M.; Herna'ndez, A.; Molina, L.; Velasco, J.; Dl'az, R. and Moriyo'n, I. (1994): Evaluation of serological tests for diagnosing Brucella melitensis infection in allergic sheep. J. Clin. Microbiol. 32: 1835-1840.

Dabdoob, WA. and Abdulla, ZA. (2000): A panel of eight tests in the seroodiagnosis and Immunilogical evaluation of acute Brucellosis. Eastern Mediterranean Health journal; 6: 304-312.

Debbarh, H.S.; Cloeckaert, A.; Zygmunt, M.S. and Dubray, G. (1995): Identification of seroreactive Brucella melitensis cytosoluble proteins which discriminate between antibodies elicited by infection and Rev.1 vaccination in sheep. Vet. Microbiol. 44: 37-48.

Gabriela Hernández-Mora; Charles A. Manire; Rocío GonzálezBarrientos; Elías Barquero-Calvo; Caterina Guzmán-Verri; Lydia Staggs; Rachel Thompson; Esteban Chaves-Olarte and Edgardo Moreno (2009): Serological Diagnosis of Brucella Infections in Odontocetes. Clin Vaccine Immunol.June; 16(6): 906-91512

Di'az, R.; Toyos, J.; Salvo, M.D.; Ferna'ndez, L.L.; Alonso-Urmementa, Moriyo'n, B.I. and Dorronsoro, I. (1984): Studies on the polysaccharide B and native hapten of Brucella melitensis and Yersinia enterocolitica serotype 9. Dev. Biol. Stand. 56: 213-220. 
Di'az, R.; Toyos, J.; Salvo, M.D. and Pardo, M.L. (1981): A simple method for the extraction of polysaccharide B from Brucella cells for use in the radial immunodiffusion test diagnosis of bovine brucellosis. Ann. Rech. Vet. 12: 35-39.

Di'az-Aparicio, E.; Arago'n, V.; Marı'n, C.; Alonso, B.; Font, M.; Moreno, E.; Pe'rez-Ortiz, S.; Blasco, J.M.; Di'az, R. and Moriyo'n, I. (1993): Comparative analysis of Brucella serotype $\mathrm{A}$ and $\mathrm{M}$ and Yersinia enterocolitica O:9 polysaccharides for serological diagnosis of brucellosis in cattle, sheep, and goats. J. Clin. Microbiol. 31: 3136-3141.

Di'az-Aparicio, E.; Marı'n, C.; Alonso-Urmeneta, B.; Arago'n, V.; Pe'rez-Ortiz, S.; Pardo, M.; Blasco, J.M.; Dl'az, R. and Moriyo'n, I. (1994): Evaluation of serological tests for diagnosis of Brucella melitensis infection of goats J. Clin Microbiol. 32: 1159-1165.

Elberg, S.S. (1981): Rev. 1 Brucella melitensis vaccine. Part II. 19681980, Vet. Bull. 51: 67-73.

Falade, S. (1978): A comparison of three serological tests in the diagnosis of caprine brucellosis. Res. Vet. Sci. 24: 376-377.

Fensterbank, R.; Verger, J.M. and Grayon, M. (1987): Conjunctival vaccination of young goats with strain Rev. 1 . Ann. Rech. Vet. 18: 397-403.

Jime'nez de Bagüe's; M.P.; Marı'n, C.M.; Blasco, J.M.; Moriyo'n, I. and Gamazo, C. (1992): An ELISA with Brucella lipopolysaccharide antigen for the diagnosis of $B$. melitensis infection in sheep and for the evaluation of serological responses following subcutaneous or conjunctival $B$. melitensis strain Rev.1 vaccination. Vet. Microbiology. 30: 233-241.

Jones, L.M.; Garcia-Carrillo, C. and Alton, G.G. (1973): Brucella melitensis Rev.1and Brucella abortus 45/20 vaccines in goats: serological tests. Am. J. Vet. Res. 34: 199-202.

Moreno, E.; Speth, S.L.; Jones, L.M. and Berman, D.T. (1981): Immunochemical characterization of Brucella lipopolysaccharides and polysaccharides. Infect. Immun. 31: 214-222.

Nicoletti, P. (1990): Vaccination, p. 283-299. In K. Nielsen and J.R. Duncan (ed.) Animal brucellosis. CRC Press, Inc., Boca Raton, Fla. 
Nielsen, K.H.; Wright, P.F.; Kelly, W.A. and Cherwonogrodzky, J.H. (1988): A review of enzyme immunoassay for detection of antibody to Brucella abortus in cattle. Vet. Immunol. Immunopathol. 18: 331-347.

Peterfy, F.; Kuusela, P. and Makela, O. (1998): Affinity requirements for antibody assays mapped by monoclonal antibodies. J. Immunol. 130: 1809-1813. 25.

Plommet, M. and Fensterbank, R. (1984): La vaccination antibrucellique administree par voi conjontivale. Dev. Biol. Stand. 56:681-687.

Waghela, S.; Wandera, J.G. and Wagner, G.G. (1980: Comparison of four serological tests in the diagnosis of caprine brucellosis. Res. Vet. Sci. 28: 168-171.

Wright, P.F. and Nielsen, K.H. (1990): Current and future serological methods, p. 305-320. In L.G. Adams (ed.), Advances in brucellosis research. Texas A\&M University Press, College Station.

Zundel, E.; Verger, J.M.; Grayon, M. and Michel, R. (1992): Conjunctival vaccination of pregnant ewes and goats with $B$. melitensis Rev. 1 vaccine: safety and serological responses. Ann. Rech. Vet. 23: 177-188. 
Assiut Vet. Med. J. Vol. 56 No. 125 April 2010 